\title{
Expectativas quanto ao Futuro: Reprovação e Expulsão da Escola de Adolescentes Estudantes e Socioeducandos
}

\section{Expectations about Future: Failure and Expulsion from School in Adolescent Students and Law-offenders}

\author{
Kátia Simone da Silva Silveira (orcid.org/0000-0001-5726-4769)1 \\ Jana Gonçalves Zappe (orcid.org/0000-0002-4452-643X)2 \\ Samara Silva dos Santos (orcid.org/0000-0002-7171-5113) ${ }^{3}$ \\ Ana Cristina Garcia Dias (orcid.org/0000-0003-2312-3911)4
}

\begin{abstract}
Resumo
Este artigo objetivou comparar dois grupos de adolescentes - escolares e socioeducandos - quanto à reprovação e expulsão da escola e às expectativas quanto ao futuro. Participaram do estudo 146 adolescentes (73 estudantes de escolas públicas e 73 socioeducandos) com idades entre 12 e 21 anos $(M=16,53$; DP=1,56). Os participantes responderam ao Questionário Juventude Brasileira, que avalia fatores de risco e proteção ao desenvolvimento. Os dados foram analisados por meio do programa estatístico Statistical Package for the Social Sciences (SPSS), sendo efetuadas análises descritivas e inferenciais. Os resultados indicaram que os socioeducandos apresentam escolaridade mais baixa, vivenciaram mais a experiência de reprovação e expulsão da escola e têm expectativas mais baixas com relação ao estudo e ao trabalho. Conclui-se que o contexto escolar é um importante ambiente de promoção do desenvolvimento saudável durante a adolescência, sendo crucial no estabelecimento de ações preventivas e de enfrentamento da transgressão juvenil. Destarte, o acesso e a permanência dos adolescentes na escola, assim como o estabelecimento e a manutenção de expectativas positivas quanto ao futuro acadêmico e profissional, devem ser encorajados.
\end{abstract}

Palavras chaves: Adolescência. Escola. Projeto de vida.

\begin{abstract}
This study aimed to compare failure and expulsion from the school and expectations about the future in two groups of adolescents - students and law-offenders. Participants were 146 adolescents (73 students from public schools and 73 law-offenders) aged between 12 and 21 years $(M=16.53, S D=1.56)$. Participants answered the

\footnotetext{
${ }^{1}$ Faculdade Integrada de Santa Maria, Santa Maria, Brasil. E-mail: katia.silveira@fisma.com.br.

${ }^{2}$ Universidade Federal de Santa Maria, Santa Maria, Brasil. E-mail: janazappe@hotmail.com.

${ }^{3}$ Universidade Federal de Santa Maria, Santa Maria, Brasil. E-mail: silvadossantos.samara@gmail.com.

${ }^{4}$ Universidade Federal do Rio Grande do Sul, Porto Alegre, Brasil. E-mail: anacristinagarciadias@gmail.com.
} 
Brazilian Youth Questionnaire, which assesses risk and protective factors for development. The data were analyzed through the statistical program Statistical Package for the Social Sciences (SPSS), and descriptive and inferential analyses were performed. Results showed that the law-offender adolescents had lower education levels, experienced more school failure and expulsion, and had lower expectations regarding study and work. It is concluded that the school setting is an important environment for promoting healthy development during adolescence, being a crucial context for preventive and coping actions related to juvenile offense. Access and permanence of adolescents in school, as well as the establishment and maintenance of positive expectations for educational and professional future should be encouraged.

Keywords: Adolescence. School. Life project. 


\section{Introdução}

No Brasil, as ações preventivas e de enfrentamento à prática de atos infracionais por adolescentes são asseguradas por meio da Doutrina de Proteção Integral, que garante a crianças e adolescentes o reconhecimento como sujeitos de direitos e a consideração pela sua condição peculiar de desenvolvimento (Lei n. 8.069, 1990). A partir disso, em termos preventivos, preconiza-se a promoção do desenvolvimento saudável por meio da garantia da convivência familiar e comunitária e do acesso a serviços de saúde, educação, assistência social, cultura e lazer. Em termos de enfrentamento, ao adolescente que comete atos infracionais podem ser aplicadas medidas socioeducativas, as quais têm caráter eminentemente educativo e visam à garantia de direitos com o objetivo de promover a cidadania e oferecer condições para a superação do conflito com a lei por meio da oferta de oportunidades educativas, profissionalizantes e de qualificação para o trabalho (Lei n. 8.069, 1990; Brasil, 2015).

A prática de atos infracionais por adolescentes é um problema complexo e multifatorial que, especialmente em razão das consequências pessoais e sociais que acarreta, tem sido foco de estudos que buscam identificar os fatores de risco e de proteção a que estão associados (Bazon, Silva \& Ferrari, 2013; Brasil, 2015; Formiga, 2010; Hein, 2004; Maruschi, Estevão \& Bazon, 2014; Silveira, Maruschi \& Bazon, 2012). A partir dessa perspectiva, compreende-se que o desenvolvimento humano é um processo que resulta da interação entre fatores de risco e de proteção, sendo que os fatores de risco podem ser características, variáveis ou eventos que, quando presentes, tornam mais provável o desenvolvimento de algum problema ou distúrbio, enquanto os fatores protetores podem ser definidos como influências que modificam, melhoram ou alteram respostas pessoais frente a adversidades ou fatores de risco (Luthar, Cicchetti \& Becker, 2000; Poletto \& Koller, 2008; Rutter, 1985).

Recentemente, foi realizada uma investigação com adolescentes brasileiros que cometeram atos infracionais utilizando de forma exploratória um instrumento canadense que contempla oito fatores de risco para a prática infracional, os quais foram identificados por meio de uma meta-análise de estudos sobre o tema (Maruschi et al., 2014). Esses fatores de risco incluem: a) infrações anteriores; b) situação familiar; c) educação e emprego; d) relação com pares; e) uso de álcool e outras drogas; f) tempo livre e recreação, g) personalidade e comportamento; e h) atitudes e orientação. Os resultados do estudo demonstraram que os fatores de risco mais predominantes foram tempo livre e recreação, educação e emprego, situação familiar e relação com pares, revelando que as dimensões de risco mais importantes são as consideradas contextuais, envolvendo família, pares, comunidade e inserção social. As dimensões individuais (personalidade, comportamento, atitudes e orientação) não apresentaram relação significativa, demonstrando que aspectos mais amplos do ambiente no qual o indivíduo está inserido são 
fundamentais para o seu desenvolvimento saudável.

Entre os fatores de risco contextuais para a prática de atos infracionais por adolescentes, destaca-se a importância do contexto escolar, que pode atuar como fator de risco ou de proteção, dependendo da relação do adolescente com a escola. O contexto escolar pode ser um fator de proteção importante quando está presente na vida dos adolescentes, pois promove a socialização e auxilia o adolescente a ter melhores perspectivas para o futuro, sobretudo quando o estudante percebe positivamente sua relação com a escola e apresenta um bom desempenho (Alves, Zappe, Patias \& Dell'Aglio, 2015; Lucero, Barrett \& Jensen, 2015; Nunes, Pontes, Silva \& Dell'Aglio, 2014). No entanto, aspectos desfavoráveis como a reprovação escolar e a percepção negativa da escola podem desmotivar o adolescente e levá-lo à evasão escolar, à diminuição de suas expectativas quanto ao futuro, à adoção de comportamentos de risco e à associação com pares que também assumem condutas de risco, entre elas a prática de atos infracionais (Alves et al., 2015; Borba, Lopes \& Malfitano, 2015; Chapman, Buckley, Sheehan, Shochet \& Romaniuk, 2011; Lemos, 2010; Nardi, 2010; Nunes et al., 2014; Rudasill, Reio Jr., Stipanovic \& Taylor, 2010; Silva, Cianflone \& Bazon, 2016).

Além disso, também é preciso considerar que esses fatores interagem de formas complexas e se influenciam mutuamente, de forma que, por exemplo, é preciso reconhecer a influência do papel da escola e especificamente das expectativas dos professores nas expectativas quanto ao futuro dos próprios adolescentes, e vice-versa. Com relação a isso, estudo conduzido por Santos et al. (2014) identificou que professores do Ensino Médio representam o adolescente como despreocupado com o futuro, sendo o aluno ideal aquele que se preocupa com o futuro. Trata-se de um dilema que pode ser superado com o desenvolvimento de ações que incentivem a preocupação com o futuro nos adolescentes e contribuam para que os professores atuem como participantes ativos nesse processo.

Algumas pesquisas demonstram que a prática de atos infracionais por adolescentes está relacionada com diversos problemas acadêmicos, como baixa escolaridade, histórico de repetência e de expulsão escolar, desmotivação e evasão escolar (Bazon et al., 2013; Davoglio \& Gauer, 2011; Gallo \& Williams, 2008; Lemos, 2010; Maruschi et al., 2014; Sanabria \& Rodríguez, 2010; Silva et al., 2016). Pesquisa realizada em Porto Alegre com 83 adolescentes do sexo masculino com idades de 13 a 19 anos que cumpriam medida socioeducativa privativa de liberdade identificou que a maioria dos adolescentes estava cursando entre a $4^{\mathrm{a}}$ e $6^{\mathrm{a}}$ série do Ensino Fundamental (60,5\%), 67\% dos adolescentes já haviam reprovado até três vezes e apenas $12 \%$ dos adolescentes nunca tinham reprovado (Davoglio \& Gauer, 2011). Outro estudo qualitativo realizado por Bazon et al. (2013) na cidade de Ribeirão Preto, com seis adolescentes do sexo masculino com idades entre 14 e 18 anos que cumpriam medida socioeducativa de semiliberdade ou liberdade assistida, revelou que 
cinco dos seis adolescentes pesquisados já haviam reprovado pelo menos uma vez, sendo que esses adolescentes cometeram todos os delitos pelos quais estavam cumprindo a medida socioeducativa durante o período de evasão escolar. No estudo de Maruschi et al. (2014), a maioria dos adolescentes participantes da pesquisa que haviam cometido ato infracional estava evadida da escola, e também foi identificado que, à medida que aumenta o nível de risco para a prática de atos infracionais, diminui a frequência às aulas e o nível de escolaridade.

Em conjunto, esses resultados parecem indicar que os problemas escolares são anteriores à prática de atos infracionais por adolescentes, sendo um aspecto de fundamental importância na condução de estudos sobre o tema (Bazon et al., 2013; Borba et al., 2015; Davoglio \& Gauer, 2011; Maruschi et al., 2014; Muller Barboza, Oliveira, Santos \& Paludo, 2009; Zappe \& Ramos, 2010). Além disso, é necessário considerar outras consequências dos problemas escolares, como diminuir expectativas em relação aos estudos em decorrência de dificuldades acadêmicas prévias à prática de atos infracionais, o que também acarreta um rebaixamento das expectativas quanto à inserção no mercado de trabalho (Muller et al., 2009; Silveira, Machado, Zappe \& Dias, 2015; Zappe \& Ramos, 2010).

As oportunidades em instituições de ensino e no mercado de trabalho têm sido marcadas por dificuldades para os adolescentes que apresentam comportamentos normativos, em função da carência de vagas e das limitações de acesso (Sobrosa, Santos, Oliveira, \& Dias,
2014), de forma que tais dificuldades podem ser ainda maiores para os adolescentes em conflito com a lei, uma vez que obstáculos culturais e econômicos somam-se às adversidades relacionadas ao envolvimento com a prática de atos infracionais (Ritter, 2010). Algumas dessas dificuldades foram descritas em estudo realizado com orientadoras educacionais de escolas frequentadas por adolescentes que estavam cumprindo medidas socioeducativas. Entre tais dificuldades, destaca-se a insuficiência de estratégias pedagógicas que atendam às especificidades desses adolescentes, a relação precária entre atores do atendimento socioeducativo e a escola, a desmotivação para o efetivo acompanhamento pedagógico dos adolescentes nas escolas e a falta de uma compreensão de que o ambiente escolar deve possibilitar o desenvolvimento integral dos estudantes (Seabra \& Oliveira, 2017).

A esse respeito, é preciso considerar que os adolescentes que cometem atos infracionais são vítimas de um sistema de exclusão, pois seu histórico de vida revela uma série de dificuldades no acesso à educação, à saúde, ao lazer e ao emprego. As possibilidades de estudo, crescimento profissional e inserção no mercado de trabalho são difíceis ou impossíveis para muitos jovens, contribuindo para o envolvimento com atos infracionais pela falta de oportunidades e chances sociais (D'aroz, 2013; Jacobina \& Costa, 2007; Shanahan, Mortimer \& Krüger, 2002).

Diante disso, compreende-se que a educação é um aspecto fundamental da ação socioeducativa, pois é um importante fator de proteção para prevenir a ocorrência de atos 
infracionais na adolescência, como também é um aspecto central da execução das medidas socioeducativas. Essas questões adquirem complexidade quando se considera o contexto escolar no interior de instituições de privação de liberdade, em decorrência das maiores dificuldades relacionadas ao desenvolvimento escolar identificadas nos casos de conflito com a lei, além de caraterísticas da própria institucionalização. Para superar essas dificuldades, Aguiar (2009) defende que a força da educação nos espaços de privação de liberdade deve partir da crença no humano e no seu potencial de mudança, buscando não apenas ampliar a oferta de educação dos espaços prisionais e socioeducativos, mas garantir a qualidade dessa oferta. Com relação a isso, o autor sugere a ampliação da discussão e da pesquisa do tema da educação em espaços de privação de liberdade, com ênfase em abordagens interdisciplinares na universidade e em institutos de pesquisa, o que poderá oferecer subsídios concretos que permitam avaliar e monitorar as políticas públicas vigentes.

A qualificação da Educação nos espaços de privação de liberdade também envolve a flexibilização da organização curricular, considerando as especificidades do contexto e a presença de estudantes em diferentes estágios de escolarização. Nesse sentido, Onofre (2015) sugere o desenvolvimento do trabalho pedagógico a partir das histórias de vida, dos interesses e dos saberes que os alunos têm, de forma que os conteúdos necessários são aqueles capazes de utilização na vida cotidiana e de promover a autonomia.

Partindo das questões apresentadas a respeito das relações entre a escola, a prática de atos infracionais por adolescentes e a intervenção educativa na socioeducação, este estudo abordou aspectos escolares em dois grupos de adolescentes, um grupo de estudantes e outro de socioeducandos, buscando avaliar a presença de reprovação e expulsão na trajetória escolar, e as expectativas quanto ao futuro nos dois grupos de adolescentes.

\section{Método}

\section{Participantes}

O estudo foi realizado utilizando os bancos de dados derivados das pesquisas "Juventude brasileira: investigando o contexto escolar na cidade de Santa Maria" e "Juventude brasileira: um estudo dos fatores de risco e proteção em adolescentes que cumprem medida socioeducativa". A amostra foi dividida em dois grupos que envolveram 146 adolescentes do sexo masculino com idade entre 12 e 21 anos $(\mathrm{M}=16,53 \mathrm{DP}=1,56)$. O Grupo 1 (G1) foi composto por 73 adolescentes do sexo masculino, estudantes de escolas públicas, escolhidos aleatoriamente. O Grupo 2 (G2) foi composto de 73 adolescentes do sexo masculino que cumpriam medida socioeducativa de internação, semiliberdade e liberdade assistida.

\section{Instrumentos}

O instrumento utilizado para a coleta de dados foi o Questionário da Juventude Brasileira (Versão Fase II - Dell'Aglio, Koller, CerqueiraSantos \& Colaço, 2011), cuja versão original tem 77 questões para identificar os comportamentos 
de risco, fatores de risco e fatores de proteção presentes em diferentes contextos que envolvem a vida dos adolescentes. Para os adolescentes do G1, foi utilizada a versão original, e para os adolescentes do G2 foi utilizada uma versão reduzida, com 47 questões, elaborada a partir de estudo prévio com socioeducandos que identificou dificuldades destes para responder integralmente ao instrumento original (Nardi, 2010).

Para responder aos objetivos deste artigo, foram utilizadas as informações presentes no instrumento relativas a informações socioeconômicas, escolaridade, reprovação, expulsão da escola e expectativas quanto ao futuro. A presença de reprovação e expulsão foi avaliada por meio de questões com opção de resposta em formato dicotômico $(0=$ não, 1=sim) e de frequência de reprovação e expulsão da escola. As expectativas quanto ao futuro foram avaliadas por meio da Escala de Expectativas quanto ao Futuro - versão reduzida adaptada de Günther e Günther (1998), presente no instrumento. A escala tem nove itens relativos às chances que $\mathrm{O}$ adolescente considera ter de: concluir o ensino médio, entrar na universidade, ter um emprego que garanta boa qualidade de vida, ter uma casa própria, ter um trabalho que dará satisfação, ter uma família, ser saudável a maior parte do tempo, ser respeitado na comunidade e ter amigos que darão apoio. As respostas foram em formato Likert de cinco pontos.

\section{Procedimentos e considerações éticas}

$$
\text { O estudo seguiu todas as }
$$

recomendações éticas para pesquisas com seres humanos estabelecidas na resolução n. 466/12 (Conselho Nacional da Saúde, 2012). Os projetos de pesquisa foram aprovados pelo Comitê de Ética em Pesquisa da Universidade Federal de Santa Maria sob os números: 0239.0.243.000.11, Protocolo: $23081.012818 / 2011.89 .11$ 16408513.2.0000.5346, número do parecer 306.036. Foi efetivado o contato com as instituições de atendimento socioeducativo e com as escolas, solicitando autorização para realização do estudo.

Depois dessa etapa, os adolescentes foram convidados a participar do estudo e foram esclarecidos de que sua participação seria voluntária, sendo garantido o sigilo de suas informações pessoais, assim como a possibilidade de desistirem da participação a qualquer momento. Os adolescentes que aceitaram participar assinaram o Termo de Consentimento Livre e Esclarecido (TCLE). Além disso, nas escolas, os pais também foram consultados para autorizar a participação de seus filhos. Os participantes puderam contar com os pesquisadores caso houvesse alguma necessidade de apoio durante ou depois da aplicação do questionário.

A composição do G1 foi realizada de forma aleatória, por conglomerados (sorteio das escolas públicas do município em que a coleta foi realizada). Foram selecionadas apenas escolas que tivessem turmas a partir do sétimo ano, devido à idade mínima para responder ao questionário, ou seja, 13 e 14 anos (Dell'Aglio et al., 2011). Destas, foram sorteadas 47 escolas e duas turmas em cada uma delas para participar 
do estudo. Foi considerado o número total de alunos matriculados no Ensino Fundamental e Ensino Médio de escolas públicas de Santa Maria, no ano de 2012, com uma margem de erro estabelecida de 4\% (Barbetta, 2001). Assim, foram aplicados os questionários em todas as escolas que aceitaram participar do estudo, totalizando 15 instituições, localizadas em diferentes regiões da cidade pesquisada, conferindo abrangência ao perfil socioeconômico dos participantes. A amostra total do estudo incluiu 454 adolescentes e jovens (12 a 20 anos) de ambos os sexos (210 meninos e 244 meninas), dos quais foram selecionados aleatoriamente, por meio de programa estatístico, os 73 participantes do sexo masculino que foram considerados no presente artigo.

Para acessar o G2, foi realizado contato com a administração central da Fundação de Atendimento Socioeducativo do Rio Grande do Sul (Fase-RS) e logo depois com os locais para cumprimento de medida socioeducativa em Santa Maria com privação de liberdade (CaseSM), semiliberdade (CAS-SM) e liberdade assistida (Cededica). A amostra desta pesquisa incluiu 75 adolescentes de ambos os sexos (73 meninos e 2 meninas), os quais aceitaram o convite para participação na pesquisa e apresentaram condições de entender e responder adequadamente ao instrumento utilizado. O G2, considerado no presente estudo, inclui os 73 adolescentes do sexo masculino presentes na amostra do estudo maior.

A aplicação do instrumento durou em média 60 minutos nas escolas e foi aplicado de forma coletiva. Já no contexto da socioeducação, os questionários foram aplicados em duplas ou individualmente e o tempo variou entre 50 minutos e 1 hora e 30 minutos. A aplicação mais individualizada no contexto socioeducativo foi adotada em função das características das instituições e da disponibilização de auxílio aos adolescentes, caso apresentassem dificuldades para compreender e responder às questões, seguindo indicações de estudo prévio com essa população (Nardi, 2010).

A opção pela amostra do sexo masculino deu-se pelo fato de que a presença de meninos no contexto socioeducativo é muito superior à presença de meninas, uma vez que a prática de atos infracionais é mais frequente em adolescentes do sexo masculino (Brasil, 2015; Davoglio \& Gauer, 2011; Nardi, Jahn \& Dell'Aglio, 2014).

\section{Análise dos dados}

Os dados foram analisados por meio do programa estatístico Statistical Package for the Social Sciences (SPSS), sendo efetuadas análises descritivas (médias, desvios padrão, frequências) e inferenciais (teste Qui-Quadrado e teste t de Student). Exploraram-se possíveis associações entre reprovações e expulsões escolares e as expectativas quanto ao futuro de ambos os grupos de adolescentes. As análises estatísticas levaram em consideração o padrão para a significância estatística de probabilidade de 5\%. 


\section{Resultados e discussão}

As análises das informações sociodemográficas indicaram algumas diferenças entre os dois grupos: a maioria dos adolescentes do $G 1$ tinha entre 15 e 18 anos $(78,1 \%, n=57)$, aproximadamente $90 \%(n=66)$ eram solteiros e nenhum deles tinha filhos. No G2, grande parte dos adolescentes tinha idade entre 16 e 18 anos (73,5\%, $\mathrm{n}=53), 68,5 \%(\mathrm{n}=52)$ eram solteiros $\mathrm{e}$ aproximadamente $90 \% \quad(n=65)$ não tinham filhos. Apesar da semelhança na faixa etária dos adolescentes dos dois grupos, foram identificadas diferenças quanto à escolaridade, sobretudo no sentido de uma maior defasagem entre os socioeducandos, o que pode ser observado detalhadamente na Tabela 1. A maioria dos adolescentes do G1 está frequentando o Ensino Médio (75,3\%), enquanto a maioria dos adolescentes do G2 frequenta entre o $6^{\circ}$ e $8^{\circ}$ ano do Ensino Fundamental (68.5\%), sendo que apenas 10,9\% eram estudantes do Ensino Médio.

Tabela 1. Características quanto ao nível de escolaridade

\begin{tabular}{|c|c|c|c|}
\hline & Escolaridade & $\mathbf{N}$ & $\%$ \\
\hline \multirow{5}{*}{ G1 (escola) } & $8^{a}$ Série ou $9^{\circ}$ ano & 16 & 21,9 \\
\hline & $1^{\circ}$ ano Ensino Médio & 22 & 30,1 \\
\hline & $2^{\circ}$ ano Ensino Médio & 23 & 31,5 \\
\hline & $3^{\circ}$ ano Ensino Médio & 10 & 13,7 \\
\hline & Não responderam & 2 & 2,7 \\
\hline \multirow{8}{*}{ G2 (socioeducação) } & $4^{a}$ Série & 7 & 9,6 \\
\hline & $5^{a}$ Série & 4 & 5,5 \\
\hline & $6^{a}$ Série & 21 & 28,8 \\
\hline & $7^{a}$ Série & 15 & 20,5 \\
\hline & $8^{\text {a }}$ Série ou $9^{\circ}$ ano & 14 & 19,2 \\
\hline & $1^{\circ}$ ano Ensino Médio & 5 & 6,8 \\
\hline & $2^{\circ}$ ano Ensino Médio & 3 & 4,1 \\
\hline & Não responderam & 4 & 5,5 \\
\hline
\end{tabular}

Fonte: Elaborada pelas autoras.

Além de apresentarem escolaridade mais baixa, os adolescentes do G2 também revelaram maior experiência de reprovação e de expulsão da escola, pois $93,1 \%$ dos adolescentes do G2 já foram reprovados e 59,2\% já foram expulsos, enquanto $69,9 \%$ dos adolescentes do
G1 já foram reprovados e apenas 5,5\% já foram expulsos. Com relação à frequência de vezes em que foram expulsos ou reprovados, os adolescentes do G2 também apresentaram índices mais elevados, o que pode ser observado na Tabela 2. 
Tabela 2. Frequência de reprovação e expulsão da escola

\begin{tabular}{lccc}
\hline \multicolumn{1}{c}{ Variáveis } & $\begin{array}{c}\text { Escola (G1) } \\
\text { Média (DP) }\end{array}$ & $\begin{array}{c}\text { Socioeducação (G2) } \\
\text { Média (DP) }\end{array}$ & $p$-valor \\
\hline $\begin{array}{l}\text { Quantas vezes você já foi } \\
\text { reprovado? }\end{array}$ & $1,32(1,11)$ & $2,70(1,50)$ & $<0,001^{*}$ \\
$\begin{array}{l}\text { Quantas vezes você já foi } \\
\text { expulso? }\end{array}$ & $0,04(0,20)$ & $1,50(2,76)$ & $<0,001^{*}$ \\
\hline
\end{tabular}

*Teste Qui-Quadrado

Fonte: Elaborada pelas autoras.

Esses resultados confirmam a relação entre a prática de atos infracionais por adolescentes e a presença de problemas acadêmicos, incluindo baixa escolaridade, reprovação e expulsão escolar (Bazon et al., 2013; Davoglio \& Gauer, 2011; Gallo \& Williams, 2008; Lemos, 2010; Maruschi et al., 2014; Sanabria \& Rodríguez, 2010; Silva et al., 2016). Essa relação pode ser explicada no sentido de que os problemas acadêmicos, provavelmente prévios ao desenvolvimento do conflito com a lei, acabam por desencadear a desmotivação do adolescente pelos estudos, promovendo a evasão escolar e gerando falhas no processo de socialização e de conquista da cidadania. Assim, considera-se que o desenvolvimento escolar é um aspecto de fundamental importância na formulação de estratégias de prevenção à prática de atos infracionais por adolescentes, de forma que $o$ acesso e a permanência na escola devem ser enfatizados, buscando-se o estabelecimento de uma relação positiva entre o adolescente e $\mathrm{o}$ contexto escolar (Alves et al., 2015; Bazon et al., 2013; Davoglio \& Gauer, 2011; Lucero et al., 2015; Maruschi et al., 2014; Muller et al., 2009; Nunes et al., 2014; Zappe \& Ramos, 2010). Além disso, os aspectos escolares devem receber especial atenção na formulação das ações socioeducativas, uma vez que é preciso reinserir os adolescentes em conflito com a lei no contexto escolar e incentivá-los a superar os problemas acadêmicos vivenciados, especialmente a reprovação, a baixa escolaridade, a expulsão e a evasão da escola (Muller et al., 2009; Zappe \& Ramos, 2010).

Com relação às expectativas quanto ao futuro, observou-se que os adolescentes do G1 apresentaram expectativas mais altas de concluir o ensino médio, entrar na universidade e ter um emprego que garanta boa qualidade de vida, as quais foram estatisticamente significativas. Esses resultados podem ser observados na Tabela 3 .

Tabela 3. Expectativas quanto ao futuro 


\begin{tabular}{lccccc}
\hline \multicolumn{1}{c}{ Variáveis } & Média & $\begin{array}{r}\text { G1 } \\
\text { DP }\end{array}$ & $\begin{array}{c}\text { G2 } \\
\text { Média }\end{array}$ & $\begin{array}{c}\text { DP } \\
\text { D-valor }\end{array}$ \\
\hline Concluir o Ensino Médio (segundo grau) & 4,19 & 0,91 & 3,1 & 1,26 & $<0,001$ \\
Entrar na universidade & 3,43 & 1,17 & 2,22 & 1,22 & $<0,001$ \\
Ter um emprego que me garanta boa qualidade de & & & & & \\
vida & 4,1 & 0,98 & 3,71 & 1,5 & 0,032 \\
Ter minha casa própria & 4,1 & 0,95 & 0,95 & 1,2 & 0,832 \\
Ter um trabalho que me dará satisfação & 4,13 & 0,98 & 4,18 & 1,03 & 0,778 \\
Ter uma família & 4,16 & 1,04 & 4,38 & 0,88 & 0,187 \\
Ser saudável a maior parte do tempo & 4,06 & 1,03 & 4,32 & 1,05 & 0,14 \\
Ser respeitado na minha comunidade & 4,12 & 0,93 & 4,25 & 1 & 0,437 \\
Ter amigos que me darão apoio & 4,19 & 1,05 & 4,07 & 1,2 & 0,565 \\
\hline
\end{tabular}

Fonte: Elaborada pelas autoras.

As diferenças encontradas com relação às expectativas acadêmicas (concluir o Ensino Médio e entrar na universidade) parecem estar relacionadas com os problemas escolares, em especial a baixa escolaridade e o número de reprovações e expulsões da escola, presentes de maneira mais marcante no G2, resultado que está de acordo com estudos prévios (Bazon et al., 2013; Gallo \& Williams, 2005; Muller et al., 2009; Zappe \& Ramos, 2010), mas não foi realizado nenhum teste específico, neste estudo, para verificar essa associação. Além disso, é preciso considerar que a oferta de oportunidades de acesso aos estudos, especialmente em nível universitário, ainda é deficitária, o que também colabora para o rebaixamento das expectativas quanto ao futuro acadêmico (Sobrosa et al., 2014). Isso foi evidenciado no estudo desenvolvido por Müller et al. (2009) com adolescentes que cometeram atos infracionais. Esses estudantes descrevem o prosseguimento de uma educação formal entre seus projetos de futuro, no entanto, quando questionados sobre a viabilidade de sua inserção em instituições de ensino superior, eles afirmaram que esse desejo dificilmente seria realizado em função da falta de oportunidade de acesso a todos.

As expectativas mais baixas apresentadas pelos adolescentes do G2 quanto a ter um emprego que garanta qualidade de vida também parecem refletir as dificuldades escolares que foram mais significativas para esse grupo, que apresentou escolaridade mais baixa e maior frequência de reprovações e expulsões, pois o desenvolvimento acadêmico é um importante fator para a conquista de um trabalho que garanta qualidade de vida. Embora este estudo não tenha avaliado as condições socioeconômicas dos participantes, estudos indicam que, muitas vezes, adolescentes que precisam trabalhar desde cedo e contribuir com o orçamento familiar podem acabar abandonando a escola para inserção no mercado de trabalho informal ou em subempregos, que, em geral, oferecem condições mais precárias em termos de características do trabalho e da 
remuneração, o que também contribui para o rebaixamento das expectativas de conquistar um trabalho mais digno e recompensador Jacobina \& Costa, 2007; Müller et al., 2009; Shanahan et al., 2002).

O trabalho, na maioria das vezes, é visto como algo benéfico pelos jovens em geral, pois possibilita a aquisição de ganhos financeiros e favorece a conquista de maior autonomia e liberdade em relação aos pais (Dutra-Thomé, Queiroz \& Koller, 2010; Sobrosa et al., 2014). No entanto, o exercício de trabalhos informais e de subempregos não permite a significação do trabalho como meio de satisfação, crescimento e aprendizagem, ou seja, como um modo de garantir qualidade de vida.

\section{Considerações finais}

Em conjunto, os resultados deste estudo acarretam importantes implicações para o avanço do conhecimento sobre a prática de atos infracionais por adolescentes e para a qualificação das ações de prevenção e enfrentamento dessas situações. Em termos do avanço do conhecimento, os resultados apresentados levam à construção da hipótese de que há uma relação entre problemas acadêmicos, baixas expectativas quanto ao futuro e a prática de atos infracionais na adolescência. A partir disso, considera-se que o contexto escolar pode ser um importante ambiente de proteção ao desenvolvimento saudável durante a adolescência, sendo crucial no estabelecimento de ações preventivas e de enfrentamento da prática de atos infracionais por adolescentes. Nesse sentido, ações de prevenção devem promover o acesso e a permanência dos adolescentes na escola, favorecendo $\mathrm{O}$ estabelecimento e a manutenção de expectativas positivas quanto ao futuro acadêmico $\mathrm{e}$ profissional. Do mesmo modo, ações socioeducativas devem incluir o retorno e a manutenção do adolescente na escola, estimulando a construção de expectativas positivas quanto ao estudo e trabalho.

Em termos de aplicação prática dos resultados do estudo, considera-se que é importante introduzir a temática dos projetos futuros na comunidade escolar, desenvolvendo ações que contribuam para a construção coletiva de projetos de futuro para a juventude, os quais sejam capazes de promover a inserção social, produtiva e realizadora dos alunos (Santos, Cruz \& Belém, 2014). Para isso, é importante considerar as representações que alunos, professores e outros membros da comunidade escolar têm sobre expectativas quanto ao futuro na adolescência, sobretudo no sentido de considerarem essa questão como uma construção coletiva (Santos et al., 2014).

A esse respeito, o contexto escolar no interior de instituições de privação de liberdade deve receber atenção especial, em decorrência das maiores dificuldades relacionadas ao desenvolvimento escolar identificadas na trajetória de adolescentes que cometeram atos infracionais. Destarte, considera-se fundamental desenvolver práticas educativas que possam promover o resgate do desenvolvimento escolar e o estabelecimento de uma vinculação positiva dos socioeducandos com a escola. Para isso, abordagens interdisciplinares devem ser valorizadas, uma vez que possibilitam a 
construção de uma perspectiva de atenção integral ao adolescente em conflito com a lei. Do ponto de vista institucional, a flexibilização da organização curricular deve ser considerada como um recurso que poderá contribuir com a oferta de um atendimento mais personalizado e em sintonia com as necessidades específicas dos adolescentes no contexto socioeducativo. Em conjunto, acredita-se que essas estratégias poderão levar ao desenvolvimento de um trabalho pedagógico que considere as histórias de vida, os interesses e os saberes que os alunos têm, valorizando os conteúdos úteis para a vida cotidiana, para o desenvolvimento da autonomia e da cidadania, aspectos centrais ao processo socioeducativo. Assim sendo, a educação possibilitará a construção do futuro partindo das experiências passadas, ressignificadas por meio das aprendizagens oferecidas no presente.

$$
\text { Por fim, cabe destacar algumas }
$$
limitações deste estudo, entre as quais se ressalta o caráter transversal e a utilização de um instrumento objetivo para coleta das informações. Recomenda-se que pesquisas futuras possam abordar essas questões em uma perspectiva longitudinal, esclarecendo o sentido da relação entre problemas acadêmicos e a prática de atos infracionais por adolescentes, assim como o alcance das ações preventivas e socioeducativas no sentido da superação do conflito com a lei. Além disso, pesquisas de caráter qualitativo poderão explorar os sentidos e significados que os adolescentes conferem aos aspectos investigados, incluindo as trajetórias escolares com a presença de reprovação e expulsão da escola, e as expectativas quanto ao futuro.

\section{Referências}

Aguiar, A. (2009). Educação de Jovens e Adultos privados de liberdade: perspectivas e desafios. Paidéia, 6(7), 101 121.

Alves, C. F., Zappe, J. G., Patias, N. D., \& Dell'Aglio, D. D. (2015). Relações com a escola e expectativas quanto ao futuro em jovens brasileiros. Nuances: estudos sobre Educação, 26(1), 50-65.

Barbetta, P. A. (2001). Estatística aplicada às ciências sociais. Florianópolis: Editora UFSC.

Bazon, M. R., Silva, J. L. da, \& Ferrari, R. M. (2013). Trajetórias escolares de adolescentes em conflito com a lei. Educação Revista, 29(2), 175-199.

Brasil. República Federativa do Brasil. (2015). Levantamento Anual SINASE 2013. Recuperado em 9 abril, 2016, de http://www.sdh.gov.br/noticias/pdf/lev antamento-2013.

Borba, L. O. P., Lopes, E. R., \& Malfitano, S. A. (2015). Trajetórias escolares de adolescentes em conflito com a lei: subsídios para repensar políticas educacionais. Ensaio: Avaliação e Políticas Públicas em Educação, 23(89), 937-963.

Chapman, R. L., Buckley, L., Sheehan, M. C., Shochet, I. M., \& Romaniuk, M. (2011). The Impact of School Connectedness on Violent Behavior, Transport Risk-Taking 
Behavior, and Associated Injuries in Adolescence. Journal of School Psychology, 49(4), 399-410.

D’aroz, M. S. (2013). A vez e a voz de mulberes mães com filhos e ou netos institucionalizados. Tese de doutorado em Educação na linha de cognição, aprendizagem e desenvolvimento humano da Universidade Federal do Paraná, Curitiba, Paraná.

Davoglio, T. R., \& Gauer, G. J. C. (2011). Adolescentes em conflito com a lei: aspectos sociodemográficos de uma amostra em medida socioeducativa com privação de liberdade. Contextos Clínicos, 4(1), 42-52.

Dell'Aglio, D. D., Koller, S. H., CerqueiraSantos, E., \& Colaço, V. F. R. (2011). Revisando o Questionário da Juventude Brasileira: uma nova proposta. In D. D. Dell'Aglio \& S. H. Koller (Eds.). Adolescência e juventude: vulnerabilidade e contextos de proteção (pp. 259-277). São Paulo: Casa do Psicólogo.

Dutra-Thomé, L., Queiroz, A. T., \& Koller, S. H. (2010). Inserção laboral juvenil: contexto e opinião sobre definições de trabalho. Paidéia, 20(46), 175-185.

Formiga, N. (2010). Pares sócio-normativos e condutas desviantes: testagem de um modelo teórico. Barbaroi, 32(1), 28-43.

Gallo, A. E., \& Williams, L. C. A. (2005). Adolescentes em conflito com a lei: uma revisão dos fatores de risco para a conduta infracional. Psicologia: Teoria e Prática, 7(1), 81-95.

Gallo, A. E., \& Williams, L. C. A. (2008). A escola como fator de proteção à conduta infracional de adolescentes. Cadernos de pesquisa, 38(133), 41-59.

Günther, I. A., \& Günther, H. (1998). Brasílias pobres, Brasilias ricas: perspectivas de futuro entre adolescentes. Psicologia: Reflexão e Crítica, 11, 191-207.

Hein, A. (2004). Factores de riesgo y delincuencia juvenil: revisión de la literatura nacional $e$ internacional. Santiago: Fundación Paz Ciudadana.

Jacobina, O. M. P., \& Costa, L. F. (2007). "Para não ser bandido": trabalho e adolescentes em conflito com a lei. Cadernos de Psicologia Social do Trabalho, 10(2), 95-110.

Kalbfleisch, M. L. (2004, March). Functional Neural Anatomy of Talent. The Anatomical Record Part B: The New Anatomist, 277(1), Retrieved November 20, 2015, from http://onlinelibrary.wiley.com/doi/10.10 02/ar.b.20010/pdf.

Lei n. 8.069, de 13 de julho de 1990. (1990). Dispõe sobre o Estatuto da Criança e do Adolescente e dá outras providências. Recuperado em 19 novembro, 2018, de http://www.planalto.gov.br/ccivil_03/L EIS/L8069.htm.

Lemos, I. T. (2010). Risco psicossocial e 
psicopatologia em adolescentes com percurso delinquente. Análise Psicológica, 28(1), 117-132.

Lucero, J. L., Barrett, C., \& Jensen, H. (2015). An Examination of Family and School Factors Related to Early Delinquency. Children \& Schools, 37(3), 165-173.

Luthar, S. S., Cicchetti, D., \& Becker, B. (2000). The Construct of Resilience: A Critical Evaluation and Guidelines for Future Work. Child Development, 71(3), 543-562.

Maruschi, M. C., Estevão, R., \& Bazon, M. R. (2014). Conduta infracional na adolescência: fatores associados e risco de reincidência. Arquivos Brasileiros de Psicologia, 66(2), 82-99.

Müller, F., Barboza, P. S., Oliveira, C. C., Santos, R. R. G., \& Paludo, S. S. (2009). Perspectivas de adolescentes em conflito com a lei sobre o delito, a medida de internação e as expectativas futuras. Revista Brasileira Adolescência e Conflitualidade, 1(1), 70-87.

Nardi, F. L. (2010). Adolescentes em conflito com a lei: percepsões sobre família, ato infracional $e$ medida socioeducativa. Dissertação de mestrado não publicada, Universidade Federal do Rio Grande do Sul, Porto Alegre, Rio Grande do Sul.

Nardi, F. L., Jahn, G. M., \& Dell'Aglio, D. D. (2014). Perfil de adolescentes em privação de liberdade: eventos estressores, uso de drogas e expectativas de futuro. Psicologia em Revista, 20(1), 116-137.

Nunes, T. G. R., Pontes, F. A. R., Silva, L. I. C., \& Dell'Aglio, D. D. (2014). Fatores de risco e proteção na escola: reprovação e expectativas de futuro de jovens paraenses. Psicologia Escolar e Educacional, 18(2), 203-210.

Onofre, E. M. C. (2015). Educação escolar para jovens e adultos em situação de privação de liberdade. Cadernos Cedes, 35(96), 239255.

Poletto, M., \& Koller, S. H. (2008). Contextos ecológicos: promotores de resiliência, fatores de risco e proteção. Estudos de Psicologia, 25(3), 405-416.

Ritter, C. (2010). O mercado de trabalho para o adolescente em conflito com a lei: a economia solidária como alternativa de inserção social em Santo Ângelo?. Dissertação de mestrado em Serviço Social não publicada. Pontifícia Universidade Católica do Rio Grande do Sul, Porto Alegre, Rio Grande do Sul, Brasil.

Rudasill, K., Reio Jr., T., Stipanovic, N., \& Taylor, J. (2010). A Longitudinal Study of Student - Teacher Relationship Quality, Difficult Temperament, and Risky Behavior from Childhood to Early Adolescence. Journal of School Psychology, 48, 389-412.

Rutter, M. (1985). Resilience in the Face of Adversity: Protective Factors and Resistance to Psychiatric Disorder. The 
British Journal of Psychiatry, 147(6), 598-611.

Sanabria, A. M., \& Rodríguez, A. F. U. (2010). Conductas antisociales y delictivas en adolescentes infractores y no infractores. Pensamiento Psicológico, 6(13), 203-217.

Santos, M. F. S., Cruz, F. M. L., \& Belém, R. (2014). Adolescentes podem ser alunos ideais?. Educação em Revista, 30(3), 173193.

Seabra, R. C. F. de F., \& Oliveira, M. C. S. L. de. (2017). Adolescentes em atendimento socioeducativo e escolarização: desafios apontados por orientadores educacionais. Psicologia Escolar e Educacional, 21(3), 639647. Recuperado em 19 novembro, 2018, de https://dx.doi.org/10.1590/2175353920170213111144.

Shanahan, M. J., Mortimer, J. T., \& Krüger, H. (2002). Adolescence and Adult Work in the Twenty-First Century. Journal of Research on Adolescence, 12(1), 99-120.

Silva, J. L. da, Cianflone, A. R. L., \& Bazon, M. R. (2016). School Bonding of Adolescent Offenders. Paidéia (Ribeirão Preto), 26(63), 91-100. Retrieved November 19, 2018, from https://dx.doi.org/10.1590/198243272663201611.

Silveira, K. S. S., Machado, J. C., Zappe, J. G., \& Dias, A. C. G. (2015). Projetos futuros de adolescentes privados de liberdade: implicações para o processo socioeducativo. Revista Teoria e Prática, 17(2), 52-63.
Silveira, M. A. S., Maruschi, M. C., \& Bazon, M. R. (2012). Risk and Protection for Adolescents Engaged in Practices of Offensive Conduct. Revista Brasileira de Crescimento e Desenvolvimento Humano, 22(3), 348-357.

Sobrosa, G. M. R., Santos, A. S. dos, Oliveira, C. T. de, \& Dias, A. C. G. (2014). Perspectivas de futuro profissional para jovens provenientes de classes socioeconômicas desfavorecidas. Temas em Psicologia, 22(1), 223-234.

Zappe, J. G., \& Ramos, N. V. (2010). Perfil de adolescentes privados de liberdade em Santa Maria/RS. Psicologia \& Sociedade, 22(2), 365-373.

Recebido em: 6/1/2018 Aprovado em: 31/1/2019 